\title{
THE ROLE OF FECAL CALPROTECTIN IN INVESTIGATING INFLAMMATORY BOWEL DISEASES
}

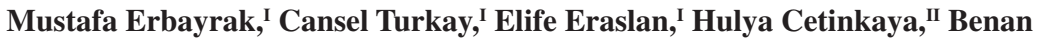 \\ Kasapoglu, ${ }^{\text {III Mehmet Bektas }}{ }^{\text {II }}$
}

doi: $10.1590 / \mathrm{S} 1807-59322009000500009$

Erbayrak M, Turkay C, Eraslan E, Cetinkaya H, Kasapoglu B, Bektas M. The role of fecal calprotectin in investigating inflammatory bowel diseases. Clinics. 2009;64(5):421-5.

INTRODUCTION: Invasive and non-invasive tests can be used to evaluate the activity of inflammatory bowel diseases.

OBJECTIVE: The aim of the present study was to investigate the role of fecal calprotectin in evaluating inflammatory bowel disease activity and the correlation of fecal calprotectin with the erythrocyte sedimentation rate and $\mathrm{C}$ reactive protein values in inflammatory bowel disease.

METHOD: Sixty-five patients affected with inflammatory bowel disease were enrolled. Twenty outpatients diagnosed with inflammatory bowel disease comprised the control group.

RESULTS: In the present study, all patients in the control group had an fecal calprotectin value lower than the cut-off point (50 $\mathrm{mg} / \mathrm{kg})$.

CONCLUSION: In conclusion, fecal calprotectin was found to be strongly associated with colorectal inflammation indicating organic disease. Fecal calprotectin is a simple and non-invasive method for assessing excretion of macrophages into the gut lumen. Fecal calprotectin values can be used to evaluate the response to treatment, to screen asymptomatic patients, and to predict inflammatory bowel disease relapses.

KEYWORDS: Inflammatory bowel diseases; Irritable bowel syndrome; Fecal calprotectin.

\section{INTRODUCTION}

Inflammatory bowel diseases (IBDs) are chronic intestinal diseases of unknown etiology that progress with periods of remission and activation. Invasive and noninvasive tests can be used to evaluate the activity of these diseases. Among those tests, the erythrocyte sedimentation rate (ESR) and $\mathrm{C}$ reactive protein (CRP) levels are neither very sensitive nor very specific. Currently, the most sensitive and specific method is endoscopy and biopsy, but it is an

\footnotetext{
${ }^{\text {I }}$ Gastroenterology Department, Fatih University Hospital - Ankara, Turkey.

II Gastroenterology Department, Ankara University Hospital - Ankara, Turkey.

III Internal Medicine Department, Fatih University Hospital - Ankara, Turkey.

Email: benankasapoglu@hotmail.com

Tel.: 90-0312-4829166

Received for publication on January 21, 2009

Accepted for publication on February on 26, 2009
}

invasive procedure. Thus, there is an increasing need for an easy, non-invasive and inexpensive method to evaluate the activity of the disease and the differential diagnosis of the disease in patients with irritable bowel syndrome (IBS).

Calprotectin, first described in 1980 by Fagertol, is released by activated neutrophils, and it accounts for more than $40 \%$ of the cytosolic proteins ofneutrophils. ${ }^{1,2}$ Elevated concentrations of calprotectin can be measured in plasma, cerebrospinal fluid, synovial fluid, urine, and feces when ongoing inflammation is present or in malignant conditions. Increased fecal calprotectin (FCP) concentrations have been found in gastric and colorectal cancers as well as in IBD. The high fecal calprotectin concentrations detected in IBD can be explained by increased turnover of leukocytes in the gut wall and increased migration of neutrophils into the gut lumen.

The aim of the present study was to investigate the role of FCP in evaluating IBD activity and remission periods and the correlation between FCP and CRP/ESR values in IBD. 


\section{MATERIALS AND METHODS}

\section{Study group}

This prospective study was carried out at Fatih University School of Medicine and Ankara University School of Medicine Department of Gastroenterology between February 2006 and February 2007. Sixty-five patients affected with IBD (14 with Crohn's Disease (CD) and 51 with Ulcerative Colitis (UC)) aged 20 to 64 years were enrolled. Twenty outpatients diagnosed with IBS according to Roma II criteria comprised the control group. ${ }^{3}$

All patients were fully informed about the objective of the study and agreed to participate. The study was approved by the Fatih University School of Medicine Ethics Committee.

\section{METHODS}

İBD surveillance, medical histories, and current treatments were all noted. Serum samples were obtained from all patients to measure the complete blood count, erythrocyte sedimentation rate (ESR), and routine biochemical measurements. $\mathrm{C}$ reactive protein $(\mathrm{CRP})$ was measured by the nephelometric method (C-Reactive Protein Reagent, IMMAGE Immunochemistry Systems Beckman Coulter, Inc., USA). Samples obtained for FCP measurement were collected in disposable plastic containers and stored within 6 hours at $-20{ }^{\circ} \mathrm{C}$ until analysis. FCP was measured using a commercial enzyme linked immunoassay (ELISA) method (Calprest, Dynex Elisa Eurospital, Trieste, Italy). The FCP results are expressed in mg of calprotectin per kilogram of wet feces within a normal reference range of $<50 \mathrm{mg} / \mathrm{kg}$.

Statistical analyses were performed using the SPSS statistical package version 11.5 for Windows. Independentsample T-tests were used for comparison of variables between groups. A $p$ value of less than 0.05 was considered as statistically significant.

\section{RESULTS}

The mean ages of the participants were $41.71 \pm 8.5$ (3055 years) in CD patients, 40.88 \pm 12.34 (20-69 years) in UC patients and $46.85 \pm 10.57$ (25-71 years) in the control group. Thirty-six of the inflammatory bowel disease patients were female and 29 were male. There were more females than males in the CD group (female/male ratio CD: 11/3, UC: $25 / 26$ ). In the control group, the female/male ratio was $15 / 5$.

The mean disease period for the patients was $4.58 \pm 0.3$ years (1-10 years) and 4.78 \pm 0.09 years (1-23 years) in the $\mathrm{CD}$ and UC groups, respectively. In the UC group, two patients had been newly diagnosed. Only three of the UC patients had a family history of IBD; one of them was a first degree relative (mother), and the other two were second degree relatives (aunt and cousin). A total of $24.6 \%$ of the patients (16 patients) were smokers, and the smoking ratio was higher in $\mathrm{CD}$ patients (7 patients or $50 \%$ in $\mathrm{CD}, 9$ patients or $17.6 \%$ in UC).

In the $\mathrm{CD}$ group, the disease was localized to the small bowel in $57.2 \%$ of the patients (8) and the ileocecal region in $42.8 \%$ of the patients (6). In $86.2 \%$ of UC patients, the disease was located in the left side of the colon, and UC had been documented in the rectosigmoid region of 9 patients in the left colon of 24 patients and in the rectum only of 11 patients. Seven patients presented with pancolitis (Table 1).

Four of the CD patients had undergone operations due to ileal obstruction and one patient displayed a perianal abscess. One of the UC patients had undergone an operation for adenocarcinoma of the rectum and another one of the UC patients demonstrated arthritis as an extra-intestinal symptom.

Investigation of the hemoglobin (hb), ESR, CRP, and FCP values of those groups revealed that ESR, CRP, and

Table 1 - Patient demographics

\begin{tabular}{lllll}
\hline & Crohn's Disease & Ulcerative Colitis & IBS (Controls) & P value \\
\hline Number of pts & 14 & 51 & 20 & P $>0.05$ \\
F/M & $11 / 3$ & $25 / 26$ & $15 / 5$ & P $<0.05$ \\
Age (mean) & 41.71 & 40.88 & 46.85 & $\mathrm{P}>0.05$ \\
Disease period (mean) & 4.58 & 4.78 & $\mathrm{P}>0.05$ \\
Smoking & $7(50 \%)$ & $9(17.6 \%)$ & $\mathrm{P}>0.05$ \\
Complication & 5 pts & 11 pts & $\mathrm{P}>0.05$ \\
Location of disease & 8 small bowel $(57.2 \%)$ & 11 rectum $(21.5 \%)$ & 9 rectosigmoid $(17.6 \%)$ & \\
& 6 ileocecal $(42.8 \%)$ & 24 left colon $(48 \%)$ & \\
& & 7 pancolitis $(12.9 \%)$ & \\
\hline
\end{tabular}


Table 2 - Hemoglobin, Erythrocyte Sedimentation Rate, C-Reactive Protein, and Fecalprotectin levels of Ulcerative Colitis, Crohn's Disease, and IBS (Controls) patients

\begin{tabular}{lllll}
\hline & Ulcerative Colites & Crohn's Disease & IBS (Controls) & puc/ pcd \\
\hline $\mathrm{Hb}(\mathrm{g} / \mathrm{dl})$ & $12.8 \pm 1.65$ & $11.61 \pm 1.39$ & $14.89 \pm 1.21$ & $0.001 / 0.001$ \\
$\mathrm{ESR}(\mathrm{mm} / \mathrm{hour})$ & $18.64 \pm 13.51$ & $34.94 \pm 25.56$ & $12.30 \pm 3.62$ & $0.043 / 0.001$ \\
$\mathrm{CRP}(\mathrm{mg} / \mathrm{l})$ & $17.53 \pm 21.51$ & $33.83 \pm 38.47$ & $4.28 \pm 3.34$ & $0.008 / 0.002$ \\
$\mathrm{FCP}(\mathrm{mg} / \mathrm{kg})$ & $264.73 \pm 37.36$ & $261.45 \pm 209.37$ & $16.72 \pm 14.61$ & $0.001 / 0.001$ \\
\hline
\end{tabular}

Puc: $\mathrm{p}$ value for the UC and control groups. Pcd: $\mathrm{p}$ value for the $\mathrm{CD}$ and control groups

FCP values were higher in the IBD patients than in the control group, while the hb level was lower in the IBD group. In particular, there was a statistically significant difference in FCP and hb levels upon comparison of the IBD patients and control group $(\mathrm{p}<0.05)$. No statistically significant differences in FCP levels were detected between $\mathrm{UC}$ and $\mathrm{CD}$ patients ( $\mathrm{p}>0.05$ ) (Table 2). Among CD patients, ESR and CRP levels were significantly higher during active disease than in remission $(\mathrm{p}<0.05)$. When we investigated the control group and patients in remission according to the Crohn's Disease Activity Index (CDAI), we found that hb levels were higher in controls, while ESR, CRP, and FCP levels were lower, and these differences were statistically significant $(\mathrm{p}<0.05)$.

Of 51 patients in the UC group, 21 were in remission according to the clinical Rachmilewitz endoscopic activity index ${ }^{4}$. Of the 32 patients with active disease, 10 were mild, 19 were moderate, and 3 were severe according to the Truelove-Witts criteria. ${ }^{5}$

Investigation of FCP levels in UC patients in remission and the control group showed that FCP levels were significantly higher in UC patients. This significant difference was probably due to the extremely high FCP levels $(>230 \mathrm{mg} / \mathrm{kg})$ in two patients. There were no significant differences in CRP and ESR levels between those two groups.

When UC patients with active disease and the control group were investigated, FCP levels in particular, as well as ESR, CRP, and UC levels were significantly higher in UC patients. Comparison of UC patients revealed that those with active disease had significantly higher ESR, CRP, and FCP levels than those in remission.

\section{DISCUSSION}

Inflammatory bowel diseases (IBDs) are chronic intestinal diseases that progress with periods of remission and activation. Although recently there is growing data regarding disease progression, diagnosis, and treatment options, we still are in need of easy, non-invasive, accurate, and inexpensive methods to estimate disease activity, determine therapy, and differentiate IBD flairs from IBS symptoms, which have been observed in 20-30\% of IBD patients. ${ }^{6,7}$ Since the correlation between laboratory data and clinical activity as assessed by endoscopy and histology is very poor in IBD, many studies are currently focused on fecal markers.

In the present study, we determined the FCP levels of UC and $\mathrm{CD}$ patients and assessed IBS patients as a control group to illustrate the role of FCP in evaluating IBD activity and remission periods. Moreover, we investigated the relationship between FCP and other markers of inflammation.

In the present study, the mean age of patients was 41.7 years in the $\mathrm{CD}$ group and 40.8 years in the UC group. The majority of patients were in their $2^{\text {nd }}-4^{\text {th }}$ decades of life. Compatible with the literature, while the number of men and women were similar in the UC group, the number of women was higher in the $\mathrm{CD}$ group. ${ }^{8,9}$ Among the $\mathrm{CD}$ patients, five of the seven smokers had active disease, but only three of the nine UC smokers presented active disease. As noted in many previous studies, smoking is linked to an increased prevalence and risk of relapse for CD. ${ }^{10,11}$

CDAI is a commonly used clinical and laboratory index for the determination of CD activity and response to treatment ${ }^{12}$. In the present study, all patients in remission according to CDAI were undergoing immunosuppressive treatment. The FCP levels were elevated in all of the patients with active disease. Many studies have previously concluded that there is no correlation between the clinical scenario and pathology in $\mathrm{CD}$, or no relationship between the CDAI and mucosal inflammation. ${ }^{13}$

Significantly higher levels of FCP were detected in the IBD group compared to the control group. In the $\mathrm{CD}$ group, very high FCP levels were determined in patients with active disease according to CDAI clinical criteria compared with patients in remission and the control group. ESR and CRP were also higher in patients with active disease, but the difference in CRP was not statistically significant. FCP levels were also significantly higher in CD patients in remission than in the control group according to CDAI. 
Leukocyte scintigraphy is the gold standard test to demonstrate inflammation, and it provides information about the localization and severity of bowel inflammation. Although this test is the proper method for differentiating functional symptoms from symptoms that are secondary to inflammation (and thus to estimate the response to treatment), the high cost and exposure to ionizing radiation have limited its use as a routine test. In a study performed by Saverymuttu et al., fecal excretion of $\mathrm{In}^{131}$-labelled granulocytes was higher in $89 \%$ of CD patients who were considered to be in remission $(\mathrm{CDAI}<150)$ than in IBS patients. ${ }^{14}$ Gaya et al. also showed a correlation between FCP and leukocyte scintigraphy in terms of determining the severity and extent of CD; in fact, FCP is more successful in showing intestinal inflammation than leukocyte scintigraphy. Moreover, there is no significant correlation between CDAI and FCP or leukocyte scintigraphy in determining disease activity. ${ }^{13}$

Roseth et al. used infliximab to treat patients with severe relapsing $\mathrm{CD}$ and found that after treatment, over a period of 3-4 weeks, FCP values decreased from 1000-2000 mg/L to $200-300 \mathrm{mg} / \mathrm{L}$, with higher values often correlating with clinically active disease. ${ }^{6}$ They found that FCP values were normalized after treatment based on both an endoscopically and histologically normal mucosal lining; furthermore, the prognosis of patients with mucosal healing was better compared to non-healing patients. Therefore, those authors suggested that mucosal healing should be a treatment goal for IBD patients. The study concluded that FCP is a significant marker in determining disease activity and response to treatment in IBD patients.

The ESR, CRP, and FCP values were significantly higher in those UC patients with clinically and endoscopically active disease than in patients in remission or in the control group. When we investigated FCP values in UC patients in the remission and control groups, we found that FCP values were significantly higher in the UC group (p: 0.015). This result may be due to the FCP values of two patients in remission that were as high as patients with active disease (230, 14-240, 44). In one of those patients, the disease period was four years and the disease was localized to the left colon; in the other patient, the disease period was fourteen years and pancolitis had been documented. In the patient with UC and pancolitis adenocarcinoma of the rectum detected via colonoscopy during the follow-up period and in the other patient in remission but with high FCP the disease is activated one month later. Although the patients were considered 'in remission', the high FCP values showed continuous mucosal inflammation and high relapse risk in the future. Patients in clinical remission and with normal
FCP values were also histologically in remission.

Tibble et al. used FCP values to predict relapses as well as to determine the necessity for IBD screening and response to treatment. They indicated that IBD patients in clinical remission with FCP values greater than $50 \mathrm{mg} / \mathrm{L}$ (in both the UC and CD groups) show clinical relapses within a few months with a sensitivity $>80 \% .^{15}$

Costa et al. performed a follow-up of 79 IBD patients in remission (CD: 31, UC: 48) for 1 year. Relapses were documented for $15 \mathrm{CD}$ and $19 \mathrm{UC}$ patients. They indicated that there was no significant difference in CRP and ESR values between patients in the relapsing and non-relapsing groups. However, they determined that, especially in the UC group, a significant difference in FCP values was present between the relapsing and non-relapsing groups. That study also showed that ESR and CRP values are not useful for estimating clinical relapses. ${ }^{\text {? }}$

In an investigation of more than 60 patients, Roseth et al. indicated that UC patients with histologically lower and higher disease activities can be distinguished simply on the basis of their FCP values. They also investigated more than 40 IBD patients with normal FCP values and determined mucosal healing in all of them. Similarly, patients in the present study with normal FCP values were those that were successfully treated and in clinical and endoscopic remission. ${ }^{6}$

Fecal calprotectin can be used as a screening test for the differential diagnosis of IBD and IBS. In the present study, all patients in the control group exhibited FCP values lower than the cut-off point $(50 \mathrm{mg} / \mathrm{kg})$. To clarify a cut off point, Tibble et al. determined $100 \%$ sensitivity and $94 \%$ specificity with a cut-off point of $30 \mathrm{mg} / \mathrm{kg}$ for FCP values in a study comprising 225 patients. In another study of 600 patients, Tibble et al. determined that patients with Roma II criteria and normal FCP values do not have IBD and indicated that there is no need for those patients to be examined radiologically or via colonoscopy. Among all IBD patients, 20-30\% may have IBS and IBS symptoms that interfere with disease activity. FCP may be used to distinguish active IBD from IBS in those patients, and high FCP values may be used as a criterion for endoscopic evaluation. On the other hand, patients with normal FCP values are most likely to have functional GIS disease.

In conclusion, fecal calprotectin was found to be a marker that is strongly associated with colorectal inflammation and to indicate organic disease. This method is a simple and noninvasive way to assess excretion of PMNs and macrophages into the gut lumen, and it is not expensive. FCP values can be used to evaluate the response to treatment when screening asymptomatic patients and to predict relapses in IBD. 


\section{REFERENCES}

1. Fagerhol MK, Dale I, Andersson T. Release and quantitation of a leukocyte derived protein (L1). Scand J Haematol. 1980;24:393-8.

2. Satoru Y,Yuichi N.Masaaki Mikami. Calprotectin (S100A8/S100A9), an inflammatory protein complex from neutrophils with a broad apoptosisinducing activity. Biol Pharm Bull. 2003;266:753-60.

3. Kruis W, Thieme C, Weinzierl M, Schüssler P, Holl J, Paulus W,

4. et al. A diagnostic score for irritable bowel syndrome: Its value in the exclusion of organic disease. Gastroenterology. 1984;87:1-7.

5. Rachmilewitz D: Coated mesalazine (5-ASA) versus sulphasalazine in the treatment of active ulcerative colitis: A randomized trial. BMJ. 1989;298:82-86.

6. Truelove SC, Witts LJ. Cortisone in ulcerative colitis. Final report on therapeutic trial. Br Med J. 1995;2:1041-8.

7. Rosent AG. Determination of faecal calprotectin, a novel marker of organic gastrointestinal disorders. Digestive and liver disease. 2003;35:607-9.

8. Costa F, Mumola MG, Ceccarelli L, Bellini M, Romano MR, Sterpi C, et al. Calprotectin is a stronger predictive marker of relapse in ulcerative colitis than in Crohn's disease. Gut. 2005;54:364-8.

9. Sands BE. Crohn's disease. Sleisenger \& Fordtran's gastrointestinal and liver disease 7 th edition, WB Saunders Company. 2002;103:2005-38.
10. Jewell DP, Ulcerative colitis. Sleisenger \& Fordtran's gastrointestinal and liver disease 7 th edition, WB Saunders Company. 2002;103:2039-67.

11. Beaugerie L, Massot N, Carbonnel F, Cattan S, Gendre JP, Cosnes J. Impact of cessation of smoking on the course of ulcerative colitis. Am J Gastroenterol. 2001; 96:2113-6.

12. Odes HS, Fich A, Reif S, Halak A, Lavy A, Keter D, et al. Effects of current cigarette smoking on clinical course of Crohn's disease and ulcerative colitis. Dig Dis Sci. 2001;46:1717-21.

13. Jewell DP, Ulcerative colitis. Sleisenger \& Fordtran's gastrointestinal and liver disease 7 th edition, WB Saunders Company. 2002;103:2039-67.

14. Gaya DR, Lyon TD, Duncan A, Neilly JB, Han S, Howell J, et al. Faecal calprotectin in the assessment of Crohn's disease activity. QJ Med. 2005 ;98:435-41.

15. Saverymutti SH. Clinical remission in Crohn's disease- Assesment using faecal Indium- 111 granulocyte excretion. Digestion. 1986;33:74-9.

16. Tibble JA, Fagerhol MK, Sigthorsson G, Bjarnason I. Use of surrogate markers of inflammation and Rome criteria to distinguish organic from nonorganic intestinal disease. Gastroenterology. 2002;123:450-60. 
